\title{
HAND-ASSISTED LAPAROSCOPIC RIGHT NEPHRECTOMY AND AUTOTRANSPLANTATION FOR TREATMENT OF RENOVASCULAR HYPERTENSION
}

Antonio Marmo Lucon, Rafael Ferreira Coelho, Jose Luiz Chambô, Anuar Ibrahim Mitre, Jose Nery Praxedes, Miguel Srougi.

\section{INTRODUCTION}

Renal autotransplantation has been described as an effective method for addressing complicated urological disorders, including debilitating ureteral injury or loss, upper ureteral malignancies, complex nephrolithiasis, loin pain hematuria syndrome, and branch or distal renal artery disease. ${ }^{1}$ Standard autotransplantation involves 2 distinct surgical procedures: nephrectomy and subsequent renal graft into the iliac fossa. The technique mimics standard allografting procedures and typically uses a separate flank incision for the live donor nephrectomy and an extraperitoneal pelvic incision for renal transplantation. Alternatively, a long midline incision from the xiphoid process to the symphysis pubis maybe used to perform the entire operation transperitoneally., ${ }^{2,3}$

In an effort to decrease the morbidity and improve the cosmetic results associated with traditional autotransplantation, we describe a laparoscopic hand-assisted approach to right nephrectomy and autotransplantation through the same handport incision in 2 patients with renovascular hypertension.

\section{CASE REPORTS}

\section{Case 1}

A 22-year-old woman with Takayasu arteritis and refractory renovascular hypertension underwent a right renal artery angioplasty and stent placement 6 months before the current hospital admission. Blood pressure control improved, allowing the use of a single antihypertensive drug instead of the 4 drugs needed before the procedure. Three months after the stent placement, the blood pressure levels started to increase. Computed angiotomography and renal arteriography showed an occluded stent and renal perfusion by collateral vessels (Figure 1A). A hand-assisted laparoscopic right

Department of Urology, Hospital das Clínicas, Faculty of Medicine, University of São Paulo, São Paulo, SP, Brazil. coelhouro@yahoo.com.br
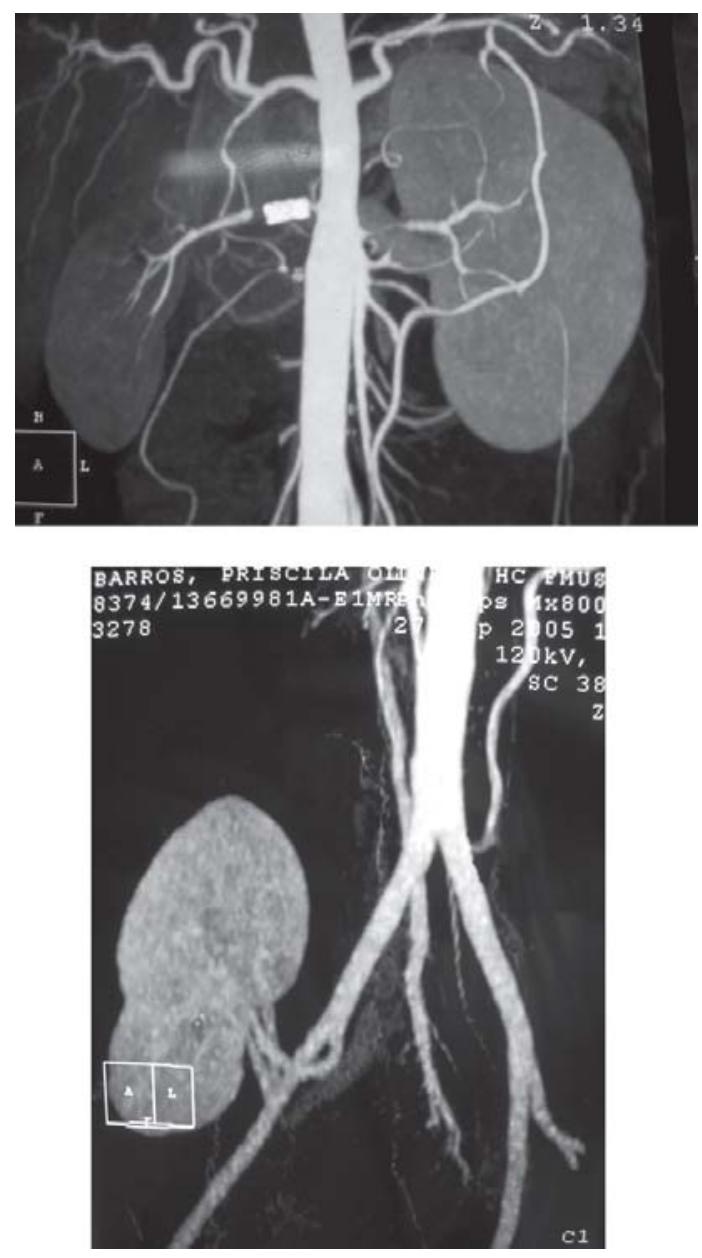

Figure 1A - Computed tomography right renal artery stent occluded and renal perfusion through collateral vessels. B - Angiotomography showing a good perfusion and normally functioning transplanted kidney.

nephrectomy for autotransplantation was planned. The patient was placed in the supine position with the right flank elevated to approximately $45^{\circ}$. A $10-\mathrm{mm}$ umbilical camera port was placed, and a right Gibson incision was made and used as a laparoscopic hand port. Subsequently, a 5-mm subxyphoid port for liver retraction, and a 10-mm right subcostal midclavicular-line working port were placed (Figure 


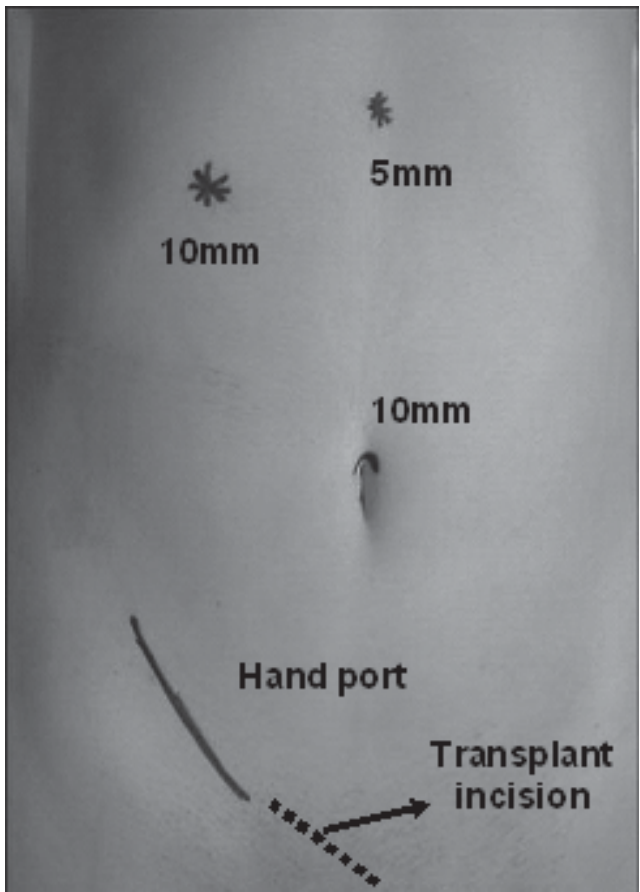

Figure 2 - Laparoscopic port positions

2). After complete mobilization of the kidney, the renal artery and vein were controlled with $1 \mathrm{Hem}-\mathrm{O}-\mathrm{Lok}^{\circledR}$ clip (Weck Closure Systems) and transected. The right ureter was not approached during the procedure. The right kidney was brought to the hand port incision and stored in an ice pack while the iliac fossa and vessels were prepared for transplantation through the Gibson incision. The patient was not repositioned for the transplantation phase. The length of the right renal vein was adequate for a successful tension-free renal transplant venous anastomosis (end-to-side to the external iliac vein). The renal-to-iliac intern-artery anastomosis was performed in an end-to-end fashion.

Hand-assisted laparoscopic nephrectomy time was 29 minutes, and total operating time was 120 minutes. Renal warm ischemia time was 2 minutes 30 seconds. Blood loss for the entire procedure was less than $300 \mathrm{cc}$. Postoperative analgesic requirements were minimal. Angiotomographic study on postoperative day 8 confirmed adequate blood flow and functioning of the transplanted kidney (Figure 1B). Blood pressure control improved after the procedure: six months after the procedure the patient required a single antihypertensive drug.

\section{Case 2}

A 17-year-old man was referred to our hospital with a diagnosis of Takayasu arteritis and renovascular hypertension since 13 years of age. He had undergone a left nephrectomy 3 years previously in another institution for treatment of renovascular hypertension secondary to left renal artery occlusion and renal atrophy. One year later, his blood pressure levels increased, and renal arteriography showed a right renal artery stenosis. He underwent a right renal artery angioplasty and stent placement with improvement of arterial hypertension. Six months after the stent placement, blood pressure control worsened, and angiotomography revealed an ostial stenosis in the right renal artery stent. (Figure 3A). Preoperative serum creatinine concentration was $1.4 \mathrm{mg} / \mathrm{dL}$. A hand-assisted laparoscopic right nephrectomy for autotransplantation was planned using the same approach outlined for case 1 . The same port locations were used, and the hand-port incision was extended inferomedially to expose the right iliac vessels. An accessory polar inferior right renal artery was identified, and an extracorporeal end-to-side anastomosis of this vessel with the right renal artery was performed while the kidney was stored in an ice pack. A renal-to-iliac intern-

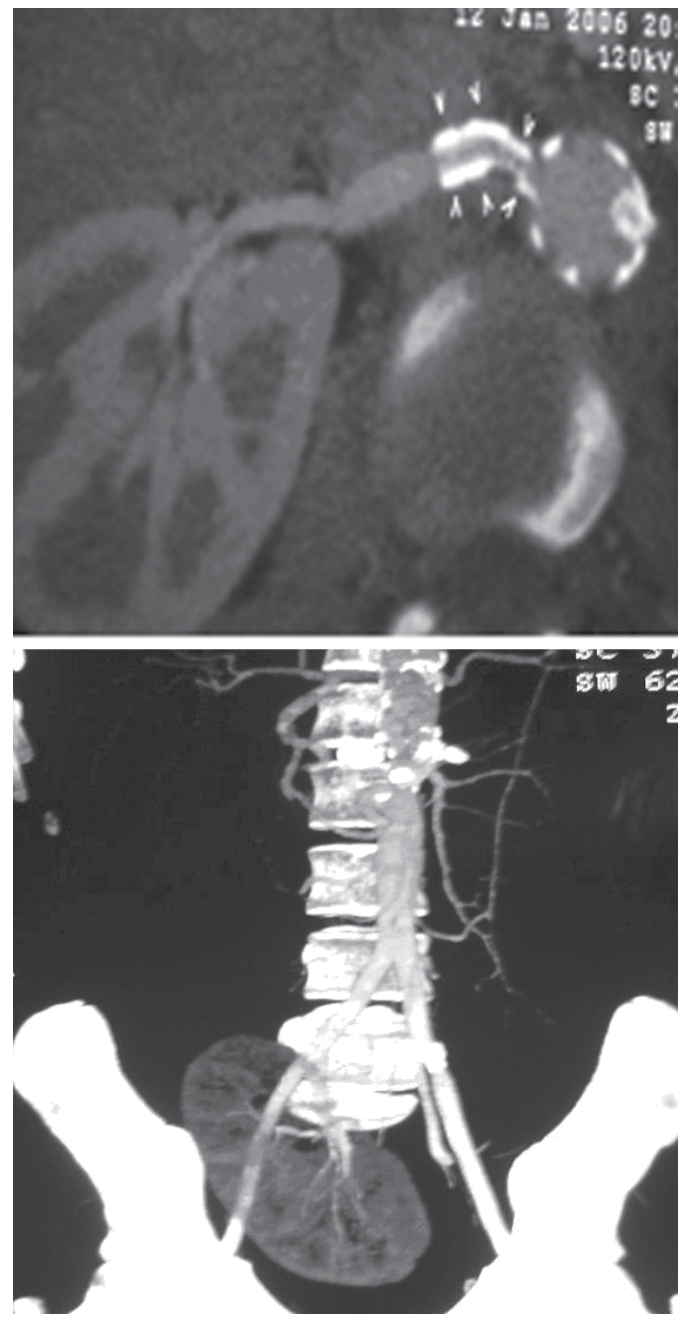

Figure 3A - Angiotomography showing an ostial stenosis in the right renal artery stent. B -Angiotomography showing a good perfusion and normally functioning transplanted kidney. 
artery anastomosis in an end-to-end fashion and a renalto-iliac extern vein anastomosis in an end-to-side fashion were performed.

Hand-assisted laparoscopic nephrectomy time was 40 minutes, and total operating time was 180 minutes. Renal warm ischemia time was 2 minutes. Blood loss for the entire procedure was less than $300 \mathrm{cc}$. An angiotomographic study and a renal scan on postoperative day 12 confirmed adequate blood flow and functioning of the transplanted kidney (Figure 3B). Blood pressure control improved after the procedure, and the patient needed just 1 antihypertensive drug 2 months postoperatively. Serum creatinine concentration at this time was $0.9 \mathrm{mg} / \mathrm{dL}$.

\section{DISCUSSION}

Renal autotransplantation was first described in 1963 for the treatment of complex ureteral injuries. ${ }^{4}$ Since them, this technique has been applied in the approach for several urological disorders, and excellent outcomes have been demonstrated at selected centers. ${ }^{5,6}$ Unfortunately, retrieving the kidney has required either a separate flank incision or extension of the pelvic transplant site. The recent explosion in the use of laparoscopic donor nephrectomy for live renal transplantation has offered an alternative method of organ procurement for transplantation, minimizing the pain, improving cosmetic aspects, and reducing recovery time. ${ }^{7}$ The largest series of laparoscopic transperitoneal nephrectomies for autotransplantation was reported by Meng and colleagues for severe ureteral-stricture disease. A purely laparoscopic transperitoneal 4-port approach with extraction of the kidney through a Gibson incision was completed in 7 patients, and the transplantation was made through the extraction incision. ${ }^{8}$ Transperitoneal laparoscopic nephrectomy with extraction through a periumbilical incision followed by transplantation through a Gibson incision ${ }^{9}$ as well as a right-sided retroperitoneal nephrectomy with kidney extraction and transplantation through the same incision have also been described. ${ }^{3}$ Troxel and colleagues ${ }^{1}$ describe a similar hand-assisted approach to renal harvesting and autotransplantation planned in such a way that the inferomedial extension of the hand-port incision provides adequate exposure of the iliac vessels for autotransplantation. The technique was applied to both left- and right-side procedures without repositioning the patient for the transplant phase. Two of the patients described in this series presented several ureteral-stricture lesions, and 1 presented loin painhematuria syndrome. The ureter was approached in all the cases. To our knowledge, our cases are the first report of a hand-assisted laparoscopic right nephrectomy and autotransplantation through the same incision for treatment of renovascular hypertension. In our cases, the ureter was not mobilized during the procedure, and no urinary tract reconstruction was necessary.

The hand-assisted approach really facilitates the renal and hilar dissection. The hand-assisted nephrectomies lasted only 29 minutes and 40 minutes in our patients, and the renal right-vein lengths obtained were adequate for a tension-free anastomosis with no vascular complications. Tactile manipulation of the tissues, easy retraction, and blunt manual dissection by intracorporeal hand can guide the surgeon in potentially complex dissections. There is no increase in morbidity because the hand-port incision was incorporated into the standard Gibson incision for the transplant phase.

\section{REFERENCES}

1. Troxel SA, Ross Jr G, Teague JL. Hand-assisted laparoscopic approach to renal harvest for autotransplantation. J Endourol. 200519:785-7

2. De Góes GM, Lucon AM. Renal autotransplantation in the treatment of hypertensive disease associated with unilateral renal artery stenosis. J Urol. 1981: 126:14.
3. Gill IS, Uzzo RG, Hobart MG, Streem SB, Goldfarb DA, Noble MJ. Laparoscopic retroperitoneal live donor right nephrectomy for purposes of allotransplantation and autotransplantation. J Urol 2000:164:15004.

4. Hardy J. High ureteral injuries: management by autotransplantation of the kidney. JAMA 1963:184:97. 
5. Bodie B, Novick AC, Rose M, Straffon RA. Long-Term results with renal autotransplantation for ureteral replacement. J Urol. 1986: 136: 1187.

6. Novick AC, Jackson CL, Straffon RA. The role of renal autotransplantation in complex urological reconstruction. J Urol. 1990: 143: 452.

7. Ratner, LE, Ciseck, LJ, Moore, RG, Cigarroa FG, Kaufman HS, Kavoussi LR. Laparoscopic live donor nephrectomy. Transplantation. 1995:60:1047
8. Meng MV, Freise CE, Stoller ML. Expanded experience with laparoscopic nephrectomy and autotransplantation for several ureteral injury. J Urol. 2003:169:1363-7.

9. Fabrizio MD, Kavoussi LR, Jackman S, Chan DY, Tseng E, Ratner LE. Laparoscopic nephrectomy for autotransplantation. Urology. 2000;55:145. 